\title{
Materials Research Society Assesses the Quality of Meetings
}

\section{Meeting Metrics}

Each time the Materials Research Society holds a meeting, the headquarters staff and volunteer members measure many numbers associated with the quality and quantity of the meeting, including the number of papers presented, number rejected, attendance at every session, number of papers per attendee, and number of posters and oral presentations. These numbers are discussed, debated, and digested by the MRS Program Committee and the MRS Council to find ways to improve future meetings. In 1994, following a Task Force chaired by Murray Gibson, the Meetings Quality Subcommittee of the Program Committee was formed, and charged with devising a useful set of metrics which focus on the overall meeting quality. Most attendees probably feel that the quality of a meeting is hard to quantify, but they know when they have been to a high-quality meeting. In general, members do not like a meeting to feel too large. This impression requires careful attention to logistics and traffic patterns. A high-quality meeting requires good scheduling, comfortable meeting rooms, good audiovisual equipment, a variety of activities, and opportunities for networking, but perhaps most importantly, a high-quality meeting must contain excellent presentations, both oral and poster. The most effective way to deliver a meeting with excellent presentations is to appoint excellent Meeting Chairs, who in turn select the best symposium organizers for each symposium topic. These choices receive very careful attention from the Council and Program Committee, and the symposium organizers receive substantial help from the headquarters staff. In addition, MRS constantly evaluates its progress on the scale of meetings quality, and here is where the meeting metrics are useful. While nobody expects a single number to capture the essence of high-quality meetings, tracking these metrics from year to year gives an objective basis for comparison which supplements the subjective impressions gathered at each meeting. This article shows recent trends in meeting size and the metrics used to monitor the quality of presentations, and describes several changes being made to further improve the quality of MRS meetings.

\section{Meeting Size}

From 1990 to 1996, the total MRS membership increased by $20 \%$ (from 10,400 to $12,400)$. The number of attendees and papers presented at the Spring and Fall

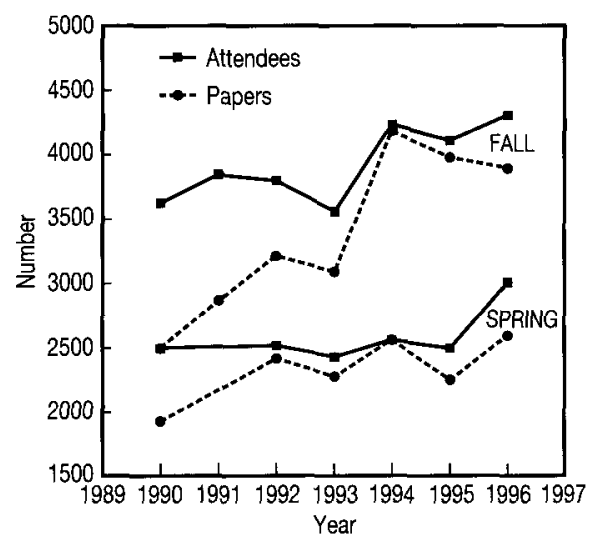

Figure 1. Number of attendees (solid line ) and number of accepted papers (dashed line) at the Fall and Spring MRS meetings from 1990 to 1996.

meetings is shown in Figure 1 for the same period. Both Spring and Fall meetings have grown in size, prompting the expansion of the Fall meeting into three Boston hotels in 1994, and filling the Spring meet- ing site in San Francisco almost to capacity. For consistency, the data for Spring 1991 are omitted because the meeting site was not in San Francisco. The configuration of three hotels in Boston has allowed the Fall meeting to grow, but the distances between meeting rooms and the decreased opportunities for encountering colleagues have prompted a reevaluation of this configuration by the Council. After extensive consideration of the options by the Long Range Planning Committee, and evaluation of member opinions by focus groups, in December 1996 the Council approved taking steps to partially move the meeting into the Hynes Convention Center in the near future. This facility, located between the Boston Marriott Hotel and the Sheraton Boston Hotel, will allow substantial consolidation of the meeting rooms plus greatly improved exhibit space.

Another way to measure the meeting size is to examine the number of attendees as a function of the total number of symposium-days. This relationship, shown in Figure 2, indicates that the number of attendees generally increases with the number of symposium-days, according to

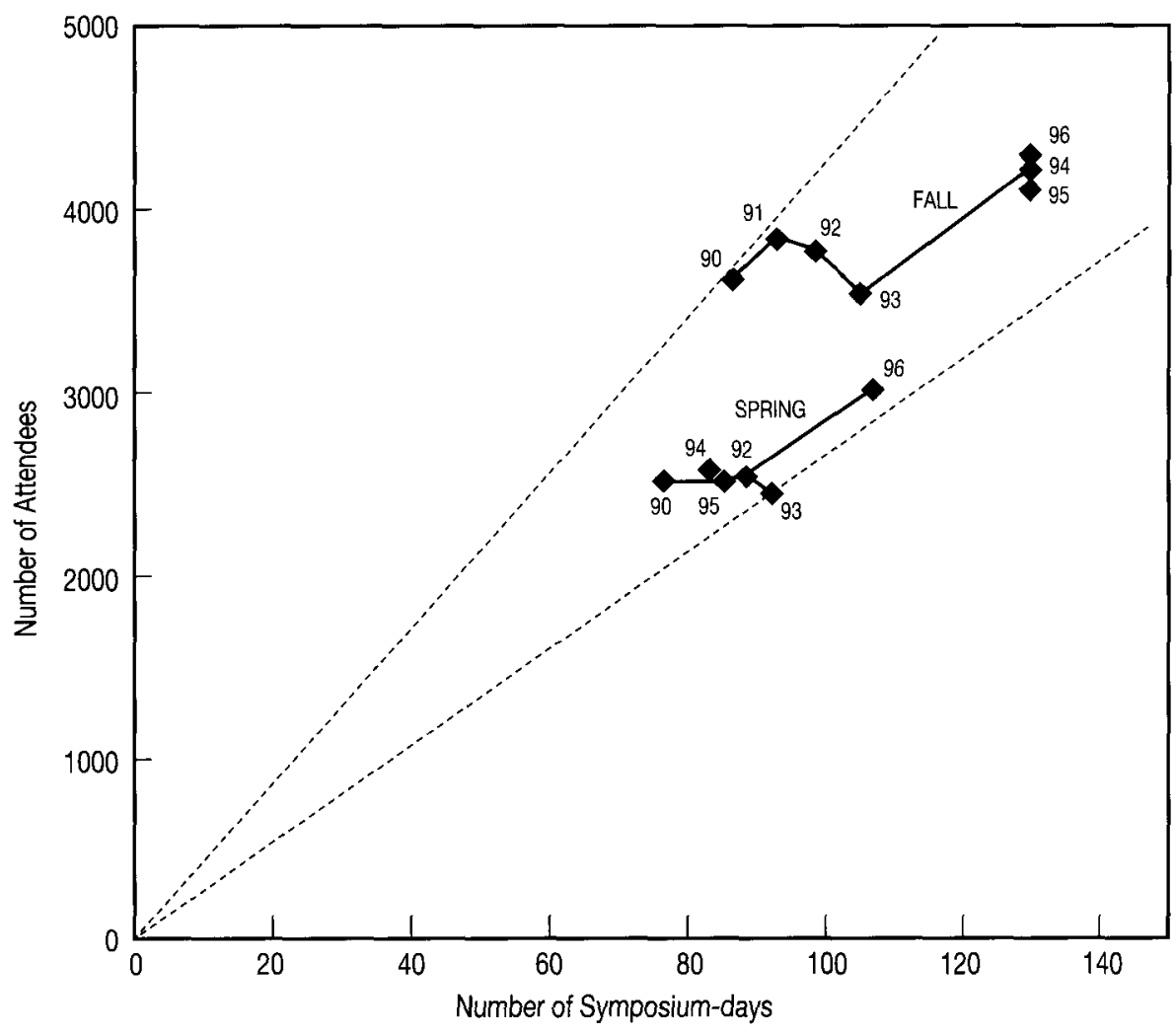

Figure 2. Number of attendees as a function of the number of symposium-days at MRS meetings from 1990 to 1996. 
a trend represented by the region between the two dashed lines. The number of symposium-days determines the overall logistics of the meeting by setting the number of meeting rooms and parallel sessions which must be scheduled. For each meeting, the Program Committee examines the needs of each symposium and works to balance the number of symposium-days allotted to large frequently presented topics as compared with providing time and space to new topics. This approach counters the tendency of large symposium topics to grow steadily larger and dominate the meetings. Instead, the intent is to continue providing a forum for new topics, while encouraging large symposium topics to become more selective in their choice of papers, and thereby become more valuable to attendees.

\section{Abstract Rejection Ratio}

Growth by itself does not guarantee improved quality, so we look at other metrics to determine whether the meeting quality is improving over time. The attendance at individual talks and symposia is carefully examined by the Program Committee to identify highly successful or unsuccessful topics. However, this information is available only after a meeting has taken place. One measure of meeting quality which we can control proactively is the degree to which the presentations undergo a rigorous selection process. It is important for authors to know that their abstracts are carefully considered and selected, rather than finding (or believing) that almost every abstract is accepted without evaluation. To monitor this process, MRS keeps track of the abstract rejection ratio, which is the ratio of the number of abstracts rejected to the number submitted. This ratio has increased substantially in the past five years, as shown in Figure 3. The abstract rejection ratio is not assumed to be a single measure of quality, but it is now receiving increased attention during the abstract selection process to ensure that the technical content of each symposium is appropriately high. Since MRS meetings consist of 25 or more distinct topical symposia, the abstract rejection ratio is not expected to be the same for all topics. For long-established topics, the ratio may be $30 \%$ or higher, while for newer topics less represented in MRS meetings, it may be less than $5 \%$. An example of the distribution of abstract rejection ratios is shown in Figure 4 for the Fall 1996 meeting, which had an overall rejection ratio of $9.7 \%$. Several symposia rejected more than $25 \%$ of submitted abstracts, while several rejected fewer than 5\%. The Program Committee has encouraged symposium organizers

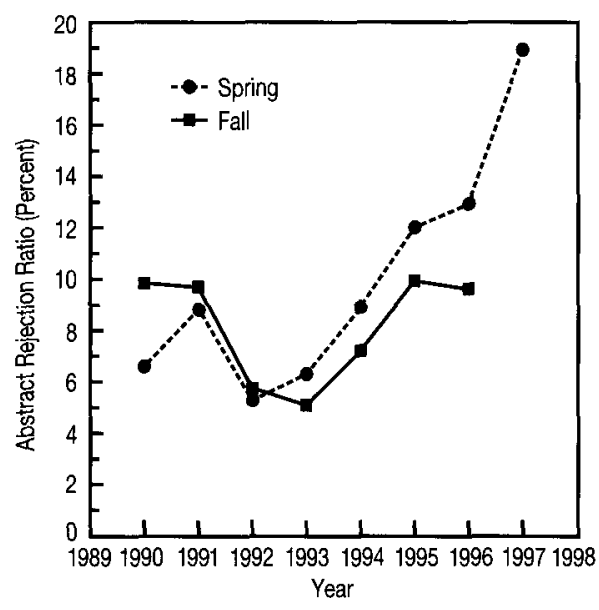

Figure 3. Abstract rejection ratio (percent) at MRS meetings from 1990 to Spring 1997.

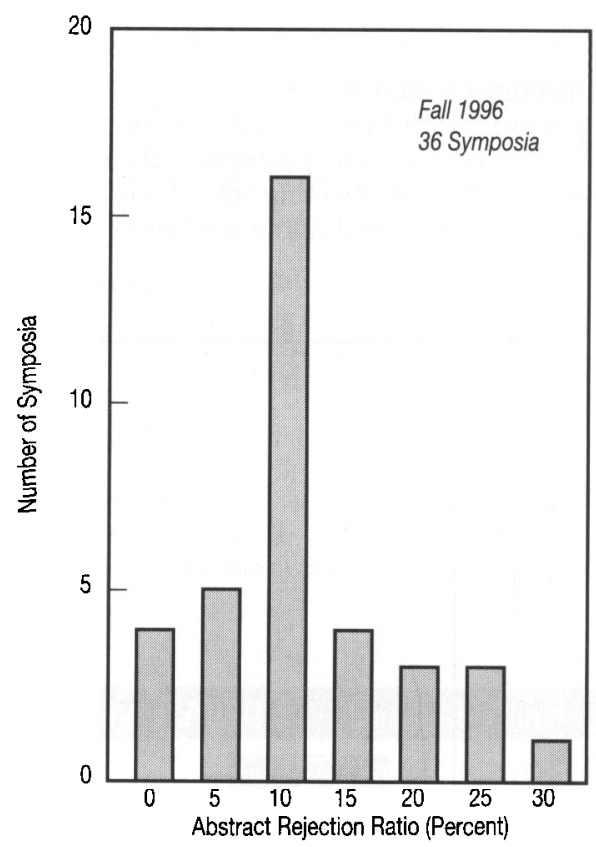

Figure 4. Distribution of abstract rejection ratios (percent) among the 36 symposia of the 1996 MRS Fall Meeting.

to increase the abstract rejection ratio to values well above the level of $5-6 \%$ of a few years ago, and this trend continues with the Spring 1997 meeting having an overall ratio around $19 \%$.

\section{Abstract Deadline}

As the number of papers has increased, the process of abstract selection has become more difficult. Symposium orga- nizers need time to select abstracts and cross-check their program schedules, but this time is limited to a few weeks between the abstract deadline and the date by which the program schedule must be finalized for printing. Complicating this process is the trend toward a large number of abstract submittals received after the abstract deadline. Authors have become accustomed to submitting late abstracts without negative consequences, symposium organizers have been reluctant to reject late submis sions of good presentations, and headquarters staff have tried to keep up with processing abstracts under the trying conditions of multiple submissions including fax and e-mail versions. The Program Committee and Meeting Chairs have decided it is time to correct this problem, and starting with the Fall 1997 meeting, the abstract deadline will be strictly observed. Adherence to the abstract deadline will allow the symposium organizers and staff to focus their attention on the important aspects of assembling a high quality meeting. There will be ample communication about the newly strict abstract deadline in upcoming meetings. The Meetings Quality Subcommittee believes that MRS members will benefit by this more sensible and equitable treatment of the abstract deadline.

\section{Summary}

This article has highlighted some of the trends in the meeting size and the abstract selection process which affect the quality of MRS meetings. Some of the changes being made to improve meeting quality include moving into the Hynes Convention Center in Boston, paying greater attention to the abstract rejection ratios, and establishing a strict abstract deadline. The Meetings Quality Subcommittee will continue to ask for attendees' opinions through surveys and focus groups, and welcomes the members' comments on issues relating to the quality of MRS meetings. Please send specific comments to the Meetings Quality Subcommittee via the author at harperj@ watson.ibm.com.

\section{Acknowledgments}

The Meetings Quality Subcommittee thanks Gail Oare, MRS Director of Meeting Activities, and the MRS headquarters staff for providing the information used in this article.

JAMES M.E. HARPER Chair, 1997 Meetings Quality Subcommittee of the MRS Program Committee 